\title{
M odelagem da variação horária da temperatura do ar em Petrolina, PE, e Botucatu, SP
}

\author{
Clóvis M. C. Ramos ${ }^{1}$, Alessandra F. da Silva ${ }^{2}$, Anderson A. da C. Sartori ${ }^{2}$, \\ Célia R. L. Zimback ${ }^{3} \&$ Luís H. Bassoi ${ }^{4}$
}

\begin{abstract}
RESU MO
A temperatura do ar é um elemento climático de interesse para diversos estudos relacionados à produção agrícola. A partir de dados de temperatura do ar de Botucatu, SP, e Petrolina, PE (diferentes classificações climáticas), medidos entre janeiro de 2004 e dezembro de 2005, foram selecionados dados pontuais com intervalos de seis horas entre cada observação para cada dia. Desta forma, foram analisados três conjuntos de dados, variando a hora do primeiro registro, utilizando a técnica de geoestatística com 0 intuito de verificar e quantificar o grau de dependência temporal entre as observações. Comprovada a dependência temporal, o método da krigagem ordinária foi utilizado a fim de modelar a variação da temperatura do ar com o intervalo de uma hora. A análise geoestatística demonstrou a dependência temporal com ajuste ao modelo esférico, em ambas as localidades, (alcance de 11,6 h para Botucatu e $12,7 \mathrm{~h}$ para Petrolina). Para ambas as localidades o horário de medida da temperatura do ar, foi fator importante na exatidão de estiamtiva da variação da temperatura. 0 s registros às $2,8,14$ e 20 h e às 4 , 10,16 e $22 \mathrm{~h}$, geraram modelos com melhor desempenho, os modelos baseados nos registros às 9,15 e $21 \mathrm{~h}$, mostraram desempenho inferior.
\end{abstract}

Palavras-chave: geoestatística, dependência temporal, elemento climático

\section{Modeling the hourly variation of air temperature at Petrolina and Botucatu, Brazil}

\begin{abstract}
AB STRACT
The air temperature is a climatic element of interest for several agricultural related studies. Hence, daily air temperature from two locations in Brazil (Botucatu and Petrolina, with different climate classification), hourly recorded from January 2004 to December 2005, were analysed to verify if $24 \mathrm{~h}$ long air temperature variation can be predicted with $6 \mathrm{~h}$ interval records. Three data set were selected, with different first time record of air temperature, and analyses performed using geoestatistics to confirm the temporal dependency of records. Then, ordinary krigging was used to model air temperature variation within $1 \mathrm{~h}$ interval, and spherical model presented the best approach, with range of $11.6 \mathrm{~h}$ for Botucatu and 12.7 for Petrolina. For both locations, the time of air temperature recording was the most importnat factor on accuracy of estimation of air temperature variation. Records at 2, 8, 14 and $20 \mathrm{~h}$ and at 4, 10, 16 and $22 \mathrm{~h}$ presented the best approaches, but for records at 9,15 and $21 \mathrm{~h}$, the approach was worst.
\end{abstract}

Key words: geoestatistics, temporal dependency, climatic element

\footnotetext{
${ }^{1}$ Doutorando em Irrigação e Drenagem/FCA-UN ESP. Fone (14) 3354-0905. Email: clovis-ramos@oi.com.br

${ }^{2}$ Mestrando em Energia na Agricultura/FCA-U NESP. Fone: (14) 3811-7169. E-mail: alefagiolli@yahoo.com.br; sartori80@gmail.com

${ }^{3}$ FCA/U N ESP, CP 237, CEP 18610-307, Botucatu, SP. Fone: (14) 3811-7165. E-mail: czimback@fca.unesp.br

${ }^{4}$ Embrapa - Semiárido, CP 23, CEP 56302-970, Petrolina, PE. Fone: (87) 3861-1711. E-mail: Ihbassoi@cpatsa.embrapa.br
} 


\section{INTRODUÇÃO}

As alterações climáticas e suas consequências para a agropecuária constituem, atualmente, uma das grandes preocupações da comunidade científica. Um dos elementos climáticos mais relacionados à produção agrícola é a temperatura do ar. A temperatura do ar afeta o comportamento germinativo das sementes. A faixa ótima de temperatura para germinação de espécies de regiões tropicais está entre 20 e $35^{\circ} \mathrm{C}$ e de regiões temperadas entre 8 e $25^{\circ} \mathrm{C}$ (Larcher, 2000). Diversos autores observaram que a variação da temperatura interfere no percentual e velocidade de germinação (Lopes \& Pereira, 2005; Lopes et al., 2005; Lima et al., 2007; Silva \& Aguiar, 2004; Steckel et al., 2004). A atividade fotossintética é dependente da temperatura do ar, uma vez que seus catalisadores expressam sua atividade máxima em determinada faixa de temperatura. Observações fenológicas da data de floração, de brotação e senescência, mantêm uma relação estreita com fatores ambientais e climáticos, o que gera melhor entendimento da fisiologia vegetal em condições naturais. A associação de séries temporais de dados fenológicos a fatores climáticos, proporciona um meio de ajudar nas previsões de impactos biológicos das mudanças climáticas futuras (Thompson \& Clark, 2006).

Desta forma, o conhecimento antecipado das condições climáticas e sua variação ao longo de um ciclo de cultivo, são significativos para a tomada de decisão e consequente intervenção, com objetivo de aumentar o rendimento da cultura. Cavalcanti et al. (2006) estimou a temperatura do ar através de modelo que utilizava coordenadas geográficas e anomalias de temperaturas da superfície do mar, para séries temporais das médias mensais de temperatura no Nordeste brasileiro. Medeiros et al. (2005), por sua vez, obtiveram estimativas da temperatura do ar mínima, média e máxima, gerando mapas temáticos para a mesma região. No estudo de séries temporais Cargnelutti Filho et al. (2006) destacam a importância de se determinar o tamanho ótimo de amostra para estimar a temperatura média mensal de uma localidade, além da variabilidade temporal e espacial. Recentemente, com o desenvolvimento e expansão dos sistemas de informações geográficas (SIG), é possível realizar a caracterização espaçotemporal de variáveis ambientais em grandes extensões territoriais (Santos et al., 2011).

A variabilidade climática anual está associada às estações do ano, Gurgel et al. (2003) observaram a relação entre a variabilidade anual e inter anual sobre a vegetação. Em latitudes médias é marcante o contraste entre as estações do ano; nos trópicos esta variabilidade é menos acentuada em termos de temperatura do ar.

Para caracterizar a variabilidade da temperatura do ar é necessário analisar sua distribuição. A variabilidade temporal pode ser estudada por meio das ferramentas da geoestatística, fundamentada na teoria das variáveis regionalizadas, em que os valores de uma variável estão relacionados à sua disposição temporal. As observações tomadas a um curto espaço de tempo se assemelham mais do que aquelas tomadas a tempos maiores (Vieira et al., 1981). Almeida et al. (2011) utilizaram essa ferramenta na análise temporal da precipitação, com melhores resultados na estimação da precipitação em comparação ao modelo de série temporal SARIMA. Estefanel et al. (1994) observaram que dias com temperatura do ar alta, ocorrem agrupados e não de forma independente. Esta temperatura do ar tende a permanecer elevada durante vários dias, até a entrada de uma massa de ar fria, sugerindo uma dependência na variação da temperatura do ar, ao longo do tempo.

A estimativa da dependência entre amostras vizinhas no espaço ou no tempo pode ser realizada através da autocorrelação, para amostragem em uma direção. Landim (2006) afirma que o semivariograma mostra a medida do grau de dependência espacial entre amostras ao longo de um suporte específico. A geoestatística estima valores em pontos não amostrados, na confecção de mapas por meio da krigagem e na modelagem de determinada variável. Desta forma, esta ferramenta pode estar ligada à obtenção de um modelo de dependência espacial ou temporal (Almeida et al., 2011). No método da krigagem, os pesos são atribuídos de acordo com a variabilidade espacial/temporal expressa no variograma.

Em estudos que exijam o conhecimento das temperaturas do ar ao longo do dia, nem sempre existem estações meteorológicas automáticas, próximas para observar esta variável climática. O tamanho da amostra (número de observações) é um importante fator para ajuste do modelo de variação horária da temperatura do ar. Ao se determinar o tamanho da amostra, em determinado local, necessária para caracterizar uma variável, é preciso estabelecer um erro máximo aceitável com um grau de confiança.

Nas estações agrometerológicas convencionais do Brasil existem registros de temperatura do ar em três horários 9, 15 e 21 h. Com base na tomada de temperatura do ar nesses horários, poderiam ser gerados modelos que estimem a variação horária da temperatura do ar durante o dia. Assim sendo, este trabalho tem por objetivo modelar a variação horária da temperatura do ar ao longo do dia para as cidades de Botucatu, SP, e de Petrolina, PE, utilizando geoestatística, a fim de conhecer os melhores horários de registros para estimar a variação da temperatura do ar ao longo de $24 \mathrm{~h}$.

\section{Material E MÉTODOS}

Os dados de temperatura do ar da cidade de Botucatu-SP foram coletados na Estação Meteorológica da Fazenda Lageado, da Faculdade de Ciências Agronômicas - UNESP, campus de Botucatu, entre janeiro de 2004 a dezembro de 2005. A fazenda se situa na latitude de $22^{\circ} 51^{\prime} \mathrm{S}$ e na longitude de $48^{\circ}$ $26^{\prime} \mathrm{W}$, com 786 metros de altitude, com temperatura média diária do mês mais frio (julho) de $17,1^{\circ} \mathrm{C}$ e a do mês mais quente (fevereiro) de $23,3^{\circ} \mathrm{C}$, e com precipitação média anual de 1314 $\mathrm{mm}$. O clima da região é do tipo Cwa, clima temperado quente (mesotérmico), de acordo com a classificação de Köppen (Cunha et al., 1999). Os dados de temperatura do ar da cidade de Petrolina, PE, foram coletados na Estação Agrometeorológica Automática de Bebedouro, pertencente à Embrapa Semiárido, Petrolina, entre janeiro de 2004 a dezembro de 2005 . A estação está situada na latitude de $09^{\circ} 09^{\prime} \mathrm{S}$ e na longitude de $40^{\circ} 22^{\prime} \mathrm{W}$, com $365,5 \mathrm{~m}$ de altitude. O clima da região é do tipo BSwh', 
segundo a classificação de Köeppen, que corresponde a uma região climaticamente árida, com período chuvoso de janeiro a abril (Teixeira et al., 2002).

Os dados de temperatura média horária do ar (T24) foram analisados utilizando-se a técnica de geoestatística. Dados pontuais com intervalos de $6 \mathrm{~h}$ entre cada observação foram selecionados para cada dia dos anos. Desta forma, foram analisados três conjuntos de dados variando a hora do primeiro registro de temperatura do ar: T2-8-14-20, com medidas às 2, 8, 14, $20 \mathrm{~h}$; T4-10-16-22 com medidas às 4,10 , 16, 22 h; e T9-15-21, com observações de temperatura às 9, $15,21 \mathrm{~h}$. Para os pontos não coletados por falha do equipamento, mantiveram-se as coordenadas do eixo horizontal e vertical sem valor de temperatura do ar. Inicialmente, verificou-se a presença de pontos discrepantes (outliers); na sequência, realizou-se uma análise descritiva dos dados para determinação da média, mediana, valor mínimo e máximo, desvio-padrão, coeficientes de variação, assimetria e curtose. A análise geoestatística foi realizada com o intuito de se verificar a existência e quantificar o grau de dependência temporal entre as observações, com base na pressuposição de estacionariedade da hipótese intrínseca, a qual é estimada pela equação abaixo, segundo Vieira et al. (1983). As variâncias foram calculadas pela Eq. 1:

$$
\gamma *(h)=\left(\frac{1}{2 N(h)}\right) \sum_{i=1}^{N(h)}\left[Z\left(x_{i}\right)-Z\left(x_{i}+h\right)\right]^{2}
$$

em que: $\gamma^{*}(\mathrm{~h})$ é a variância para um vetor $\mathrm{h}$ (dias); $\mathrm{Z}(\mathrm{x})$ e $\mathrm{Z}(\mathrm{x}+\mathrm{h})$ são os pares de observações de temperatura, separados pelo vetor h (dias); $\mathrm{N}(\mathrm{h})$ é o número de pares de valores medidos $\mathrm{Z}(\mathrm{x}), \mathrm{Z}(\mathrm{x}+\mathrm{h})$, separados por um vetor $\mathrm{h}$. Do ajuste de um modelo matemático aos valores calculados de $\gamma^{*}(\mathrm{~h})$, foram estimados os coeficientes efeito pepita $\left(\mathrm{C}_{0}\right)$, patamar $\left(\mathrm{C}_{0}+\mathrm{C} 1\right)$ e o alcance (Ao) do modelo teórico para o variograma. Os modelos foram ajustados pelo programa GS 9.0 (Robertson, 2009), considerando a menor soma do quadrado dos resíduos, o maior coeficiente de determinação $\left(\mathrm{R}^{2}\right)$ e o coeficiente de correlação obtido pelo método de validação cruzada. Comprovada a dependência temporal, utilizou-se o método da krigagem ordinária a fim de modelar a variação da temperatura do ar com o intervalo de uma hora e assim estimar os valores não medidos no tempo.

A fim de verificar a exatidão dos dados estimados foram correlacionados valores estimados com os medidos, pela regressão linear. Desta forma foram considerados os seguintes indicadores estatísticos, de precisão: coeficiente de correlação "r"; de exatidão: índice de Willmott "d" e de confiança ou desempenho "c" (Camargo \& Sentelhas, 1997). A precisão é dada pelo coeficiente de correlação que indica o grau de dispersão dos dados obtidos em relação à média, ou seja, o erro aleatório. A exatidão está relacionada ao afastamento dos valores estimados em relação aos observados. Matematicamente, esta aproximação é dada por um índice designado de concordância, representado pela letra "d" (Willmott et al., 1985). Seus valores variam de zero (para nenhuma concordância) a um (para a concordância perfeita). O índice é dado pela Eq. 2:

$$
\mathrm{d}=1-\sum(\mathrm{Pi}-\mathrm{Oi})^{2} \div \sum(|\mathrm{Pi}-\mathrm{O}|+|\mathrm{Oi}-\mathrm{O}|)^{2}
$$

donde: Pi é o valor estimado, Oi é o valor observado e O é a média dos valores observados.

O índice "c" utilizado neste trabalho indica o desempenho dos métodos, reunindo os índices de precisão " $r$ " e de exatidão “d”, expresso da seguinte forma (Eq. 3):

$$
\mathrm{c}=\mathrm{r} \times \mathrm{d}
$$

O critério adotado para interpretar o desempenho dos métodos propostos por Camargo \& Sentelhas (1997) através do valor do índice "c" é: > 0,85, ótimo; 0,76 a 0,85, muito bom; 0,66 a 0,75 , bom; 0,61 a 0,65 , mediano; 0,51 a 0,60 , sofrível; 0,41 a 0,50 , mau; d" 0,40, péssimo.

\section{RESULTADOS E DISCUSSÃO}

Nas estatísticas observadas na Tabela 1, os dados de temperatura média horária do ar em Botucatu, no periodo estudado, se mantiveram próximos da temperatura média anual da localidade, que é de $21^{\circ} \mathrm{C}$, de acordo com Cunha et al. (1999). Verifica-se uma distribuição simétrica das medidas de temperatura do ar ao longo dos dois anos de observação, fato que se repetiu em todos os horários de amostragem testados, exceto pelas observações realizadas às $2,8,14$ e $20 \mathrm{~h}$, que apresentaram valor bem diferente dos demais. É importante ressaltar que o software $\mathrm{GS}^{+}$(Robertson, 2009), utilizado para análise descritiva, adota como padrão o valor zero para distribuição mesocúrtica. O coeficiente de variação apresentou valores médios para todos os conjuntos de dados, de acordo com o critério de Warrick \& Nielsen (1980). Comparando os registros de temperatura do ar a cada $6 \mathrm{~h}$ com o conjunto de dados com 24 registros ao longo do dia (T24), constata-se que o conjunto de registros T2-8-14-20 e T4-10-18-22 tem valores

Tabela 1. Estatística descritiva da temperatura do ar em Botucatu, SP, entre janeiro de 2004 a dezembro de 2005

\begin{tabular}{lccccccccc}
\hline \multicolumn{1}{c}{ Atributos } & $\mathbf{n}$ & Média & Mediana & Mínimo & Máximo & $\mathbf{S}$ & $\mathbf{C V}$ & $\mathbf{C}_{\mathbf{s}}$ & $\mathbf{C}_{\mathbf{k}}$ \\
T24-Bot & 17283 & 20,16 & 19,96 & 6,23 & 34,12 & 4,30 & 18,50 & 0,08 & $-0,15$ \\
T2-8-14-20-Bot & 2883 & 20,21 & 19,96 & 6,23 & 34,12 & 4,29 & 18,38 & 0,17 & $-0,02$ \\
T4-10-16-22-Bot & 2881 & 20,28 & 20,09 & 6,27 & 33,59 & 4,33 & 18,73 & 0,07 & $-0,20$ \\
T9-15-21-Bot & 2165 & 21,29 & 21,11 & 7,70 & 34,03 & 4,18 & 17,51 & 0,06 & $-0,15$ \\
\hline
\end{tabular}

T24-Bot: registros de temperatura do ar horária em Botucatu $\left({ }^{\circ} \mathrm{C}\right) ;$; T2-8-14-20-Bot: medidas da temperatura do ar às $2,8,14$ e $20 \mathrm{~h}$ em Botucatu $\left({ }^{\circ} \mathrm{C}\right) ; \mathrm{T} 9-15-21$-Bot: registros de temperatura do ar às 9, 15 e 21 h em Botucatu ( $\left.{ }^{\circ} \mathrm{C}\right)$; T4-10-16-22-Bot: registros de temperatura do ar às 4, 10, 16 e $22 \mathrm{~h}$ em Botucatu ( $\left.{ }^{\circ} \mathrm{C}\right)$; $\mathrm{n}$ : número de observações; $\mathrm{S}$ : desvio padrão; $\mathrm{CV}$ : coeficiente de variação em \%; $\mathrm{C}_{\mathrm{s}}$ : coeficiente de assimetria; $C_{\mathrm{k}}$ : coeficiente de curtose 
Tabela 2. Estatística descritiva da temperatura do ar em Petrolina, PE, entre janeiro de 2004 a dezembro de 2005

\begin{tabular}{lccccccccc}
\hline \multicolumn{1}{c}{ Atributos } & $\mathbf{n}$ & Média & Mediana & Mínimo & Máximo & S & CV & $\mathbf{C}_{\mathbf{s}}$ & $\mathbf{C}_{\mathbf{k}}$ \\
T24-Pet & 17356 & 26,16 & 25,59 & 14,90 & 38,75 & 3,99 & 15,99 & 0,34 & $-0,56$ \\
T2-8-14-20-Pet & 2892 & 26,18 & 25,34 & 16,46 & 38,57 & 3,87 & 15,03 & 0,64 & $-0,31$ \\
T4-10-16-22-Pet & 2892 & 26,39 & 26,21 & 15,16 & 37,86 & 4,12 & 17,04 & 0,24 & $-0,58$ \\
T9-15-21-Pet & 2168 & 27,71 & 27,07 & 20,82 & 38,75 & 3,57 & 12,74 & 0,54 & $-0,48$ \\
\hline
\end{tabular}

T24-Pet: registros de temperatura do ar horária em Petrolina $\left({ }^{\circ} \mathrm{C}\right) ; ;$ T2-8-14-20-Pet: medidas da temperatura do ar às $2,8,14$ e $20 \mathrm{~h}$ em Petrolina $\left({ }^{\circ} \mathrm{C}\right) ;$ T9-15-21-Pet: registros de temperatura do ar às 9, 15 e $21 \mathrm{~h}$ em Petrolina $\left({ }^{\circ} \mathrm{C}\right)$; T4-10-16-22-Pet: registros de temperatura do ar às 4, 10, 16 e $22 \mathrm{~h}$ em Petrolina $\left({ }^{\circ} \mathrm{C}\right)$; $\mathrm{n}$ : número de observações; S: desvio padrão; CV: coeficiente de variação em $\%$; $\mathrm{C}_{\mathrm{s}}$ : coeficiente de assimetria; $\mathrm{C}_{\mathrm{k}}$ : coeficiente de curtose

estatísticos de média, mediana e valor mínimo que mais se aproximam do conjunto de registros $\mathrm{T} 24$; no entanto, os registros T9-15-21 e T2-8-14-20 se aproximaram de T24 nas estatisticas de valor máximo. Para os coeficientes de variação, de simetria e de curtose, os dados T4-10-16-22 e T9-15-21 ficaram mais próximos das observações T24.

Os resultados da análise descritiva da temperatura do ar em Petrolina (Tabela 2), para os dois anos de observação, mostram que a temperatura média do ar se aproxima da temperatura média anual, que é de $27^{\circ} \mathrm{C}$, segundo Moura et al. (2006). Esses dados de temperatura do ar apresentaram assimetria positiva (Cs), com média maior que a mediana, e também com maior assimetria para os conjuntos de registros T2-8-14-20 e T9-15-21. Todos os dados de temperatura do ar apresentaram distribuição de frequência platicúrtica, ou seja, com curtose menor que zero, mostrando tendência desses atributos apresentarem maior dispersão dos dados em torno da média. O coeficiente de variação apresentou valores médios para todos os conjuntos de dados, de acordo com o critério de Warrick \& Nielsen (1980). Quando se comparam as estatísticas dos conjuntos de dados com os registros horários, observa-se que os valores de média, valor minimo e coeficiente de variação, são mais próximos entre os dados T24, T2-8-14-20 e T4-10-16-22.

Comparado as duas localidades, observa-se haver maior variabilidade dos dados de temperatura do ar em Botucatu e menor variação em Petrolina, demonstrado pelo valor do coeficiente de variação e da amplitude térmica, que é maior em Botucatu. Os coeficientes de curtose e simetria mostram que, apesar da maior variabildade dos dados em Botucatu, estes estão mais simetricamente distribuidos em torno da média, enquanto para Petrolina os dados apresentam coeficiente de assimetria maior. Referidas observações corroboram com as diferenças da classificação climática das duas localidades, porém, quando se trata de temperatura média mensal, Cunha et al. (1999) obervaram a variação entre o mês mais quente e o mais frio, de apenas $7{ }^{\circ} \mathrm{C}\left(17,1 \mathrm{e} 24,3^{\circ} \mathrm{C}\right)$ em Botucatu, enquanto em Petrolina a menor variação foi de $3,5^{\circ} \mathrm{C}$ em 2000 e a maior chegou a $7,3{ }^{\circ} \mathrm{C}$ em 1984 ; já a média da variação entre o mês mais quente e o mais frio, foi de $4,6{ }^{\circ} \mathrm{C}$, no período de 1975 a 2008. De acordo com Estefanel et al. (1994), os estudos de temperatura do ar, que normalmente utilizam médias mensais ou anuais, não permitem a detectar a frequência de períodos com altas temperaturas.

Os resultados da análise geoestatística (Tabela 3) indicam que os dados de temperatura do ar apresentaram dependência temporal, ajustando-se ao modelo esférico. Os pontos localizados em tempo menor ou igual ao alcance, são mais similares e dependentes entre si, em relação ao tempo. $\mathrm{O}$ alcance (Ao), ou tempo de correlação de T24 foi de 11,6 h para
Botucatu e de 12,7 h para Petrolina, sendo os maiores alcances encontrados para os conjuntos de registros T4-10-16-22 (12,75 h) para Botucatu e T2-8-14-20 (15,00 h) para Petrolina. Os valores de tempo de correlação ratificam as observações da análise descritiva dos dados de temperatura do ar em Petrolina, que apresentaram menor variabilidade. A proximidade no valor de Ao nos modelos gerados a partir de diferentes horas de registro, comparando-a com o modelo gerado a partir dos dados horários (T24), indica maior exatidão do modelo que estima a variação da temperatura do ar ao longo do dia.

Tabela 3. Modelos e parâmetros dos variogramas da temperatura do ar para dados de Botucatu, SP, e Petrolina, PE

\begin{tabular}{llrccccc}
\hline \multicolumn{1}{c}{ Atributos } & Modelo & \multicolumn{1}{c}{ Ao } & $\mathbf{C}_{\mathbf{0}}$ & $\mathbf{C}_{\mathbf{0}}+\mathbf{C}$ & $\mathbf{R}^{2}$ & IDT & rCv \\
T24-Bot & Esférico & 11,60 & 0,810 & 16,01 & 98 & 95 & 0,91 \\
T2-8-14-20-Bot & Esférico & 12,62 & 1,930 & 16,02 & 92 & 88 & 0,77 \\
T4-10-16-22-Bot & Esférico & 12,75 & 2,070 & 16,40 & 90 & 87 & 0,77 \\
T9-15-21-Bot & Esférico & 8,56 & 0,870 & 14,81 & 77 & 94 & 0,74 \\
\hline T24-Pet & Esférico & 12,77 & 0,010 & 15,11 & 94 & 99 & 0,95 \\
T2-8-14-20-Pet & Esférico & 15,00 & 0,010 & 13,96 & 80 & 99 & 0,88 \\
T4-10-16-22-Pet & Esférico & 14,89 & 0,010 & 16,23 & 78 & 99 & 0,88 \\
T9-15-21-Pet & Esférico & 9,49 & 0,010 & 12,20 & 58 & 99 & 0,81 \\
\hline
\end{tabular}

T24-Bot: registros de temperatura do ar horária em Botucatu ( $\left.{ }^{\circ} \mathrm{C}\right)$; T2-8-14-20-Bot: medidas da temperatura do ar às $2,8,14$ e $20 \mathrm{~h}$ em Botucatu $\left({ }^{\circ} \mathrm{C}\right) ;$ T9-15-21-Bot: registros de temperatura do ar às 9,15 e $21 \mathrm{~h}$ em Botucatu $\left({ }^{\circ} \mathrm{C}\right)$; T4-10-16-22-Bot: registros de temperatura do ar às $4,10,16$ e $22 \mathrm{~h}$ em Botucatu $\left({ }^{\circ} \mathrm{C}\right)$; T24-Pet: registros de temperatura do ar horária em Petrolina $\left({ }^{\circ} \mathrm{C}\right)$; $\mathrm{T} 2$ 8-14-20-Pet: medidas da temperatura do ar às $2,8,14$ e $20 \mathrm{~h}$ em Petrolina $\left({ }^{\circ} \mathrm{C}\right)$; T9-15-21-Pet: registros de temperatura do ar às 9,15 e $21 \mathrm{~h}$ em Petrolina $\left({ }^{\circ} \mathrm{C}\right)$; T4-10-16-22-Pet: registros de temperatura do ar às $4,10,16$ e $22 \mathrm{~h}$ em Petrolina $\left({ }^{\circ} \mathrm{C}\right) ; \mathrm{Ao}$ : Alcance (horas); $\mathrm{C}_{0}$ : efeito pepita; $\mathrm{C}_{0}+\mathrm{C}$ : patamar; $\mathrm{R}^{2}$ : coeficiente de determinação múltipla do ajuste; IDT: índice de dependencia temporal; rcv: coeficiente de correlação da validação cruzada

O índice de dependência temporal (IDT), considerando-se a classificação proposta por Zimback (2001) para dependência espacial, leva em consideração a relação entre variância estruturada (C) e o valor do patamar $\left(\mathrm{C}+\mathrm{C}_{0}\right)$, na qual a dependência espacial é fraca, quando IDE $>25 \%$, moderada, quando $25 \% \leq \mathrm{IDE} \geq 75 \%$ e alta, quando IDE $>75 \%$. Adaptandose esta classificação para análise temporal da temperatura do ar, observa-se que todos os conjuntos de dados apresentaram alta dependência temporal (IDT $\geq 75 \%$ ).

O estudo do desempenho dos modelos em estimar a variação da temperatura do ar ao longo do dia, em função dos diferentes horários de registro, foi realizado com o índice de Willmot e a proposta de Camargo \& Sentelhas (1997), uma vez que a adoção do coeficiente de determinação $\left(\mathrm{R}^{2}\right)$ na definição da qualidade de um modelo não é adequada, pois o mesmo não estabelece o tipo nem a magnitude das diferenças entre um valor padrão e um valor previsto por modelos de estimativa ou outros mecanismos de medida diferentes do padrão. Observa-se, na Tabela 4, que para Botucatu os registros T2-8-14-20 e T4-1016-22 geraram modelos com melhor desempenho, não havendo 
diferença entre eles quanto ao índice c; porém verifica-se, no regitro T9-15-21, uma redução no desempenho do modelo, motivada pela redução da amostra. Para a cidade de Petrolina nota-se que os registros T2-8-14-20 e T4-10-16-22 (Tabela 5)

Tabela 4. Desempenho dos modelos em função das amostras de temperatura do ar

\begin{tabular}{lcccc}
\hline \multicolumn{1}{c}{ Modelos } & $\mathbf{d}$ & $\mathbf{r}$ & $\mathbf{C}$ & Classificação do índice "c" \\
T2-8-14-20-Bot & 0,95 & 0,92 & 0,87 & Ótimo \\
T4-10-16-22-Bot & 0,95 & 0,91 & 0,87 & Ótimo \\
T9-15-21-Bot & 0,89 & 0,85 & 0,76 & Muito bom \\
\hline T2-8-14-20-Pet & 0,96 & 0,94 & 0,90 & Ótimo \\
T4-10-16-22-Pet & 0,96 & 0,93 & 0,90 & Ótimo \\
T9-15-21-Pet & 0,85 & 0,84 & 0,71 & Bom
\end{tabular}

T24-Bot: registros de temperatura do ar horária em Botucatu $\left({ }^{\circ} \mathrm{C}\right)$; T2-8-14-20-Bot: medidas da temperatura do ar às 2, 8, $14 \mathrm{e} 20 \mathrm{~h}$ em Botucatu $\left({ }^{\circ} \mathrm{C}\right)$; T4-10-16-22-Bot: registros de temperatura do ar às $4,10,16$ e $22 \mathrm{~h}$ em Botucatu $\left({ }^{\circ} \mathrm{C}\right) ; \mathrm{T} 9-15$-21-Bot: registros de temperatura do ar às 9,15 e $21 \mathrm{~h}$ em Botucatu $\left({ }^{\circ} \mathrm{C}\right)$; T24-Pet: registros de temperatura do ar horária em Petrolina $\left({ }^{\circ} \mathrm{C}\right)$ : T2-8-14-20-Pet: medidas da temperatura do ar às 2, 8, 14 e $20 \mathrm{~h}$ em Petrolina ( ${ }^{\circ} \mathrm{C}$ ); T4-10-16-22Pet: registros de temperatura do ar às $4,10,16$ e $22 \mathrm{~h}$ em Petrolina $\left({ }^{\circ} \mathrm{C}\right): T 9-15-21$-Pet: registros de temperatura do ar às 9 , 15 e $21 \mathrm{~h}$ em Petrolina $\left({ }^{\circ} \mathrm{C}\right)$; $\mathrm{d}$ : índice de Willmot; $\mathrm{r}$ : coeficiente de correlação; C: índice de desempenho proposto por Camargo \& Sentelhas (1997)

\section{A. Botucatu, SP}

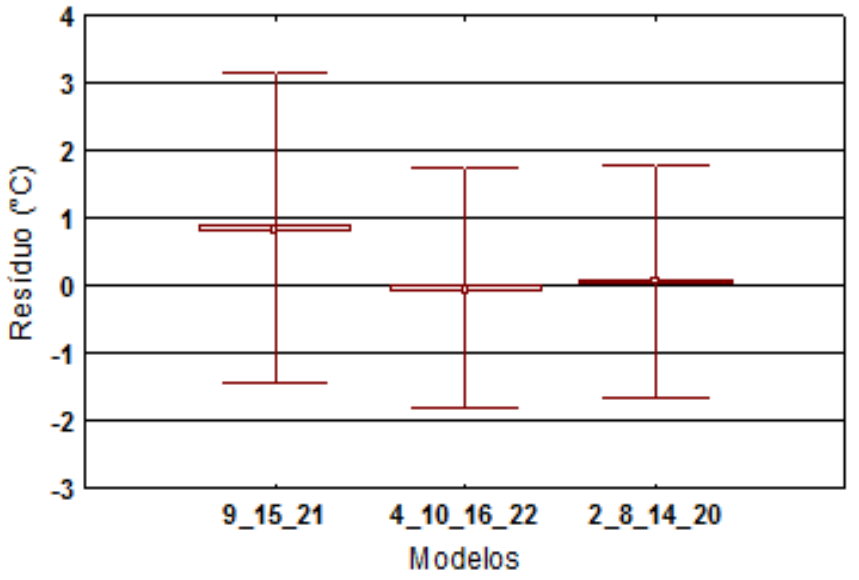

C. Botucatu, SP

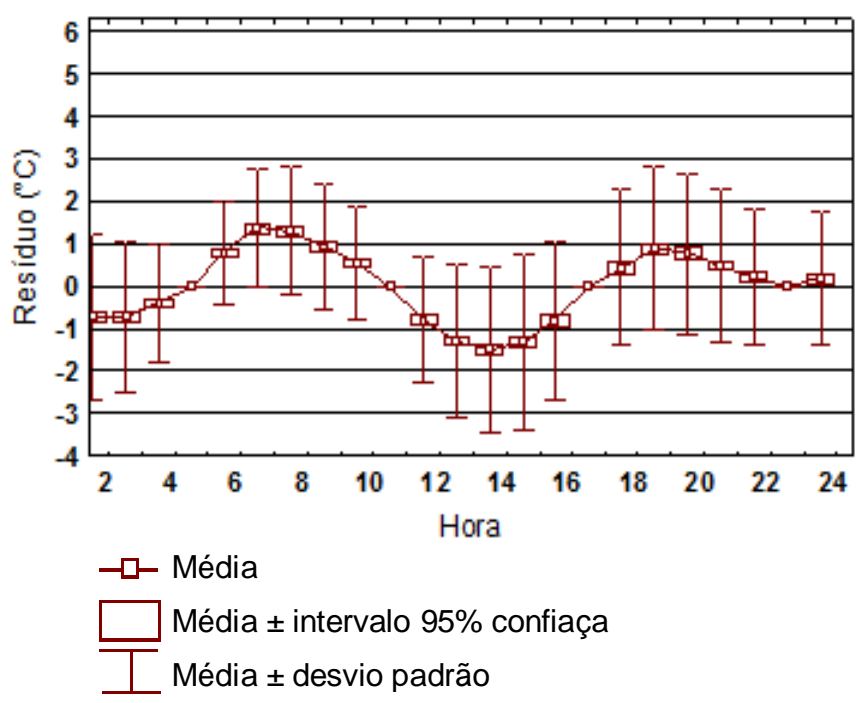

também geraram modelos com melhor desempenho, não havendo diferença entre eles quanto ao indice c, e uma redução no desempenho dos modelos gerados a partir do registro T915-21.

Comparando as duas localidades, observa-se que os modelos tiveram desempenho igual para os modelos gerados a partir dos registros T2-8-14-20 e T4-10-16-22, segundo o índice "c", proposto por Camargo \& Sentelhas (1997). Para Petrolina, o modelo gerado a partir do registro T9-15-21 indicou desempenho inferior ao que ocorreu em Botucatu, SP.

Analisando o resíduo da estimativa de cada modelo observase que os conjuntos de registros T2-8-14-20 e T4-10-16-22 obtiveram um resíduo médio que se próxima de zero, enquanto o modelo gerado pelo conjunto de registros T9-15-21 obteve resíduo acima de zero (Figura $1 \mathrm{~A}$ e 2A), mostrando tendência de superestimar a temperatura horária do ar, fato que ocorreu em ambas as localidades. Porém, quando o estudo do resíduo é detalhado para cada modelo em função das horas de estimativa, os maiores resíduos ocorrem a medida em que se distancia do

\section{B. Botucatu, SP}

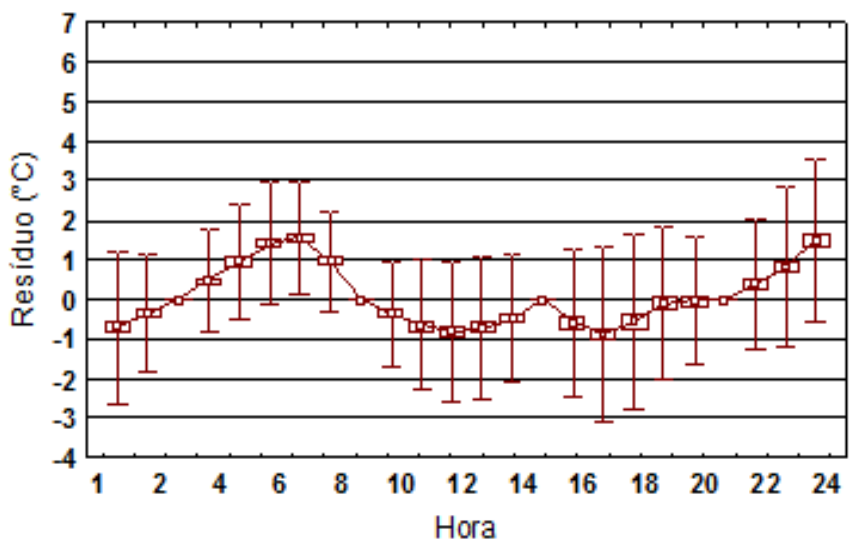

D. Botucatu, SP

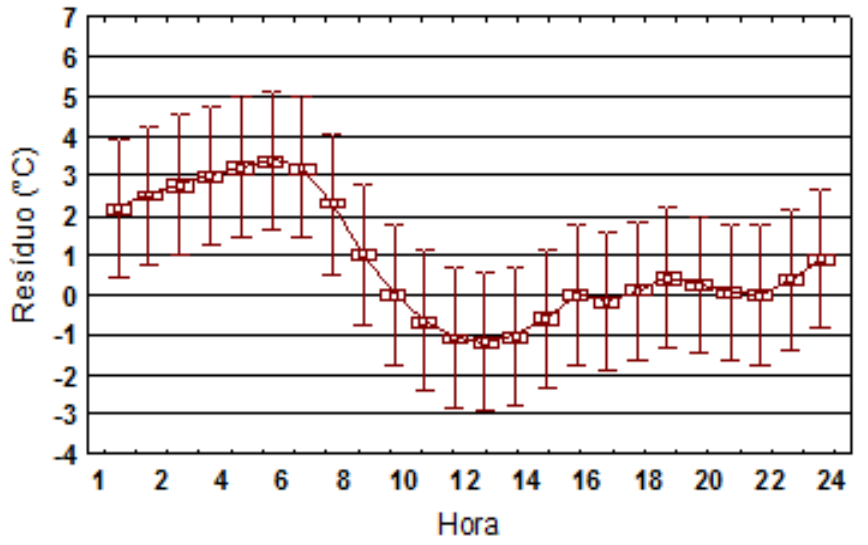

Figura 1. M odelagem da temperatura do ar para a cidade de Botucatu, SP, nos anos de 2004 e 2005. Box-plot da média dos resíduos dos modelos gerados a partir dos três conjuntos de registros estudados $(A)$; média dos resíduo da estimativa horária da temperatura do ar modelada pelo conjunto de registros T2-8-14-20 (B); média dos resíduo da estimativa horária da temperatura do ar modelada pelo conjunto de registros T4-10-16-22 (C); média dos resíduo da estimativa horária da temperatura do ar modelada pelo conjunto de registros e T9-15-21 (D) 
A. Petrolina, PE

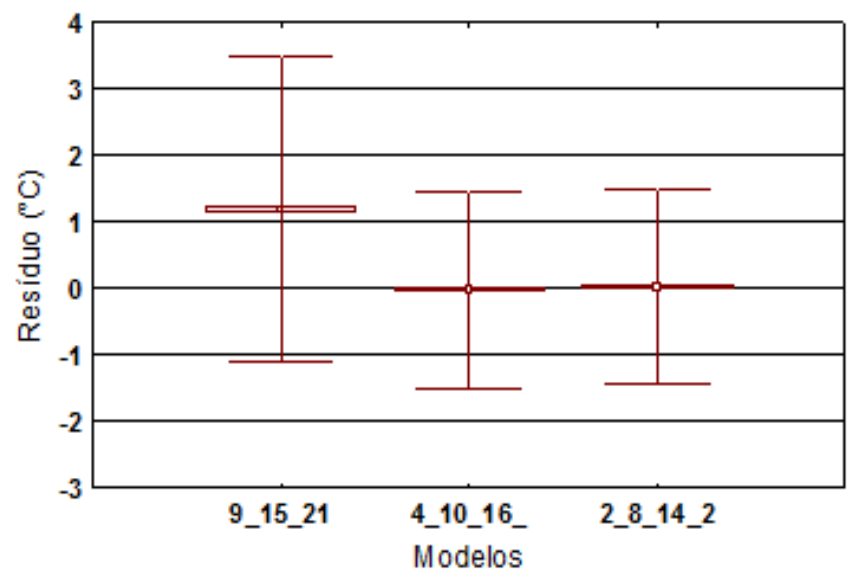

C. Petrolina, PE

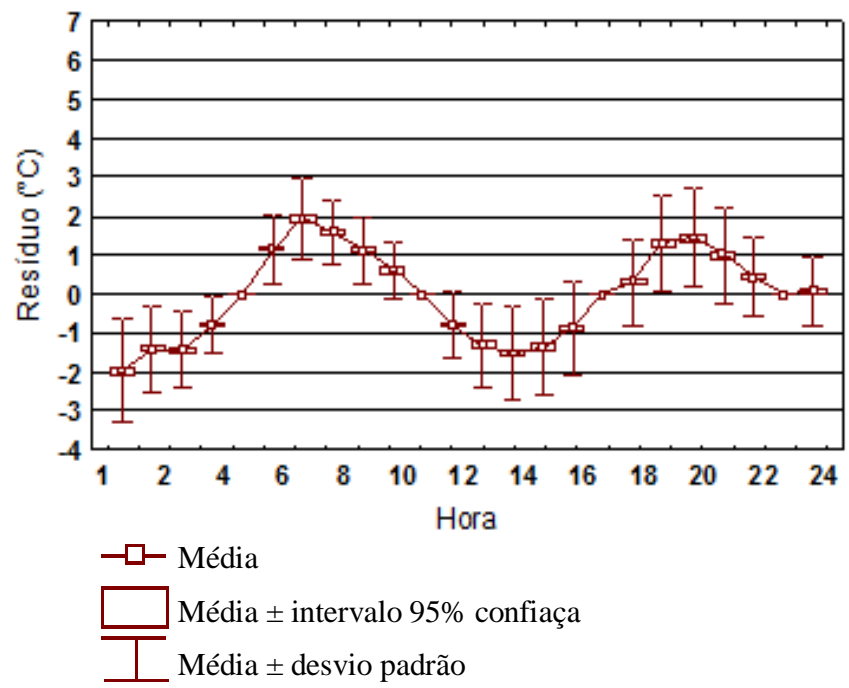

B. Petrolina, PE

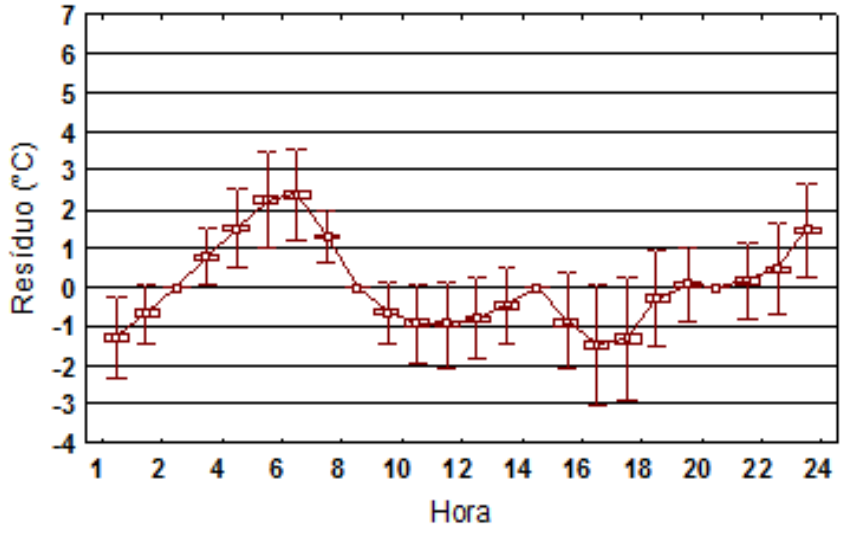

D. Petrolina, PE

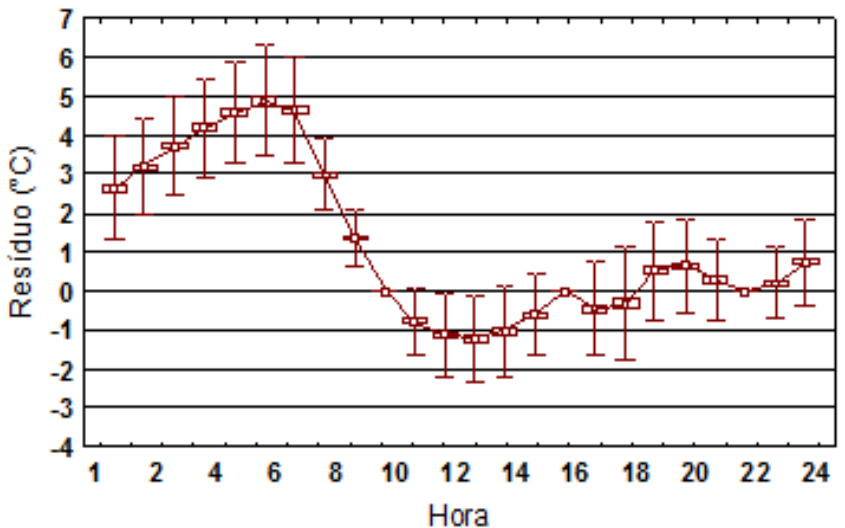

Figura 2. Modelagem da temperatura do ar para a cidade de Petrolina, Pe, nos anos de 2004 e 2005. Box-plot da média dos resíduos dos modelos gerados a partir dos três conjuntos de registros estudados (A); média dos resíduo da estimativa horária da temperatura do ar modelada pelo conjunto de registros T2-8-14-20 (B); média dos resíduo da estimativa horária da temperatura do ar modelada pelo conjunto de registros T4-10-16-22 (C); média dos resíduo da estimativa horária da temperatura do ar modelada pelo conjunto de registros e T9-15-21 (D)

ponto de amostra, com maiores variações para os modelos gerados a partir do conjunto de registros T9-15-21 (Figura 1D e 2D), e a superestimativa acontece nos horários anteriores às $9 \mathrm{~h}$ da manhã. Desta forma, as estimativas realizadas entre às 8 e 22 $\mathrm{h}$, mostraram um resíduo de $\pm 1^{\circ} \mathrm{C}$ para a cidade de Botucatu e \pm $1,5^{\circ} \mathrm{C}$ em Petrolina. Enquanto para as condições de Botucatu não há como diferenciar a exatidão dos modelos em relação aos registros das 2, 8, 14 e $20 \mathrm{~h}$ (Figura 1B) e aos registros das 4, 10, 16 e 22 h (Figura 1C), para Petrolina, observa-se que os melhores horários para medição da temperatura do ar com intuito de modelar sua variação ao longo do dia, é às $2,8,14$ e $20 \mathrm{~h}$ (Figura 2B). As medias realizadas às 9,15 e $21 \mathrm{~h}$, foi o conjunto de dados que gerou maior erro na estimativa da temperatura horária do ar, ao longo do dia, para as duas localidades estudadas.

\section{CONCLUSÕES}

1. A dependência temporal entre os dados de temperatura horária do ar ao longo do dia, existe em Botucatu, SP, e Petrolina,
PE, com variância estruturada a partir de um intervalo $12 \mathrm{~h}$ entre as observações realizadas em ambas as localidades.

2. O horário de medida da temperatura do ar junto e as condições climáticas locais, são fatores importantes que interferem na exatidão do modelo ao se estimar a variação da temperatura do ar.

3. O uso de registro de temperatura do ar em três horários $(9$, 15 e $21 \mathrm{~h}$ ) para modelagem da variação da temperatura do ar ao longo do dia, é o menos exato, quando se leva em conta os horários entre 22 e $8 \mathrm{~h}$ da manhã do dia seguinte.

\section{AGRADECIMENTOS}

Ao GEPAG - Grupo de Pesquisas Agrárias Georreferenciadas, FCA/UNESP Botucatu, pelo apoio e infra-estrutural; ao Departamento de Ciências Ambientais, FCA/UNESP Botucatu, pelos dados meteorológicos fornecidos.

A Embrapa Semiárido, pelo fornecimento dos dados de temperatura do ar de sua estação meteorológica. 


\section{LITERUTURA CITADA}

Almeida, A. Q.; Ribeiro, A.; Paiva, Y. G.; Rascon, N. Jr. L.; Lima, E. P. Geoestatística no estudo de modelagem temporal da precipitação. Revista Brasileira de Engenharia Agrícola e Ambiental, v.15, p.354-358, 2011.

Camargo, A. P. de; Sentelhas, P. C. Avaliação do desempenho de diferentes métodos de estimativa da Evapotranspiração potencial no estado de São Paulo, Brasil. Revista Brasileira de Agrometeorologia, v.5, p.89-97, 1997.

Cargnelutti Filho, A.; Matzenauer, R.; Maluf, J. R. T. Variabilidade temporal e espacial do tamanho de amostra da temperatura mínima do ar no Rio Grande do Sul, Brasil. Ciência Rural, v.36, p.1156-1163, 2006.

Cavalcanti, E. P.; Silva, V. P. R.; Sousa, F. A. S. Programa computacional para a estimativa da temperatura do ar para a Região Nordeste do Brasil. Revista Brasileira de Engenharia Agrícola e Ambiental, v.10, p.140-147, 2006.

Cunha, A. R.; Klosowski, E. S.; Galvani, E.; Escobedo, J. F.; Martins, D. Classificação climática para o município de Botucatu, SP, segundo Köppen. In: Simpósio em Energia na Agricultura, 1, Botucatu. Anais... Botucatu: FCA/UNESP, 1999, v.1, p.487-490.

Estefanel, V.; Schneider, F. M.; Buriol, G. A. Probabilidade de ocorrência de temperaturas máximas do ar prejudiciais aos cultivos agrícolas em Santa Maria, RS. Revista Brasileira de Agrometeorologia, v.2, p.57-63, 1994.

Gurgel, H. C. Variabilidade espacial e temporal do NDVI sobre o Brasil e suas conexões com o clima. São José dos Campos. Instituto Nacional de Pesquisas Espaciais (INPE), 2003. 120p. Dissertação Mestrado

Gurgel, H. da C.; Ferreira, N. J.; Luiz, A. J. B. Estudo da variabilidade do NDVI sobre o Brasil, utilizando-se a análise de agrupamentos. Revista Brasileira de Engenharia Agrícola e Ambiental, v.7, p.85-90, 2003.

Landim, P. M. B. Sobre geoestatística e mapas. Terra e Didática, v.2, p.19-33, 2006.

Larcher, W. Ecofisiologia vegetal. São Carlos: Rima, 2000. 531p.

Lima, R. V.; Lopes, J. C.; Coelho, R. I. Germinação de sementes de urucu em diferentes temperaturas e substratos. Ciência e Agrotecnologia, v.31, p.1219-1224, 2007.

Lopes, J. C.; Capucho, M. T.; Martins Filho, S.; Repossi, P. A. Influência de temperatura, substrato e luz na germinação de sementes de bertalha. Revista Brasileira de Sementes, v.27, p.18-24, 2005.

Lopes, J. C.; Pereira, M. D. Germinação de sementes de cubiu em diferentes substratos e temperaturas. Revista Brasileira de Sementes, v.27, p.146-150, 2005.
Medeiros, S. S.; Cecílio, R. A.; Melo Júnior, J. C. F.; Silva, J. L. C. Estimativas e espacialização das temperaturas do ar mínimas, medias e máximas na Região Nordeste do Brasil. Revista Brasileira de Engenharia Agrícola e Ambiental, v.9, p.247-255, 2005.

Moura, M. S. B. de; Sá, I. I. S.; Silva, T. G. F. da; Galvíncio, J. D.; Ribeiro, J. G. Variação espacial da precipitação e temperatura do ar no Submédio São Francisco. In: Congresso Brasileiro de Meteorologia, 14, 2006, Florianópolis. Anais ... Florianópolis: Sociedade Brasileira de Meteorologia, 2006.

Robertson, G. P. GS+: Geoestatistics for the environmental sciences - GS+ User's Guide. Plainwell: Gamma Desing Software, 2009. 152p.

Santos, E. H. M. dos; Griebeler, N. P.; Oliveira, L. F. C. de. Variabilidade espacial e temporal da precipitação pluvial na bacia hidrográfica do Ribeirão João Leite-GO. Engenharia Agrícola, v.31, p.78-89. 2011.

Silva, L. M. de M.; Aguiar, I. B. de. Efeito dos substratos e temperaturas na germinação de sementes de Cnidosculus phyllacanthus Pax \& K. Hoffm. (Faveleira). Revista Brasileira de Sementes, v.26, p.9-14, 2004.

Steckel, L. E.; Sprague, C. L.; Stoller, E. W.; Wax, L. M. Temperature effects on germination of nine amaranthus species. Weed Science, v.52, p.217-221, 2004.

Teixeira, A. H. de C.; Bassoi, L. H.; Costa, W. P. L. B. da; Silva, J. A. M.; Silva, E. E. G. da. Consumo hídrico da bananeira no Vale do São Francisco estimado pelo método da razão de Bowen. Revista Brasileira de Agrometeorologia, v.10, p.4550, 2002.

Thompson, R.; Clark , R. M. Spatio-temporal modelling and assessment of within-species phenological variability using thermal time methods. International Journal of Biometeorology, v.50, p.312-322, 2006.

Vieira, S. R.; Hatfield, T. L.; Nielsen, D. R.; Biggar, J. W. Geostatistical theory and application to variability of some agronomical properties. Hilgardia, v.51, p.1-75, 1983.

Vieira, S. R.; Nielsen, D. R.; Biggar, J. W. Spatial variability of field-measured infiltration rate. Soil Science Society of Americam Journal, v.45, p.1040-1048, 1981.

Warrick, A. W.; Nielsen, D. R. Spatial variability of soil physical properties in the field. In: Hillel, D. (ed.). Application of soil physics. New York: Academic Press, 1980. 385p.

Willmott, C. J.; Ackleso, S. G.; Davis, R. E.; Feddema, J. J.; Klink K. M.; Legates, D. R.; O’Donnell J.; Rowe, C. M. Statistics for the evaluation and comparison of models. Journal of Geophysical Research, v.90, p.8995-9005, 1985.

Zimback, C. R. L. Análise espacial de atributos químicos de solos para fins de mapeamento da fertilidade. Botucatu: FCA/UNESP, 2001. 114p. 